\title{
A Stable Indium-Pyridylcarboxylate Framework with Highly Selective Adsorption of Cationic Dyes and Effective Nitenpyram Detection
}

Qianqian Chu, ${ }^{\dagger}$ Bin Zhang, ${ }^{\ddagger}$ Zhipeng Yang, ${ }^{\dagger}$ Huifang Zhou, ${ }^{*}+$ Haibo Mu, ${ }^{\dagger}$

Wenyan Zhang, $\neq$ Bo Liu, ${ }^{*}$, and Yao-Yu Wang ${ }^{\ddagger}$

College of chemistry \& pharmacy, Northwest A\&F University, Yangling 712100, P.R. China

¥Key Laboratory of Synthetic and Natural Functional Molecule Chemistry of the Ministry of Education, Shaanxi Key Laboratory of Physico-Inorganic Chemistry, College of Chemistry \& Materials Science, Northwest University, Xi'an 710069, P.R. China

`E-mail:hfzhou@nwafu.edu.cn, chemliubo@nwsuaf.edu.cn.

(a)

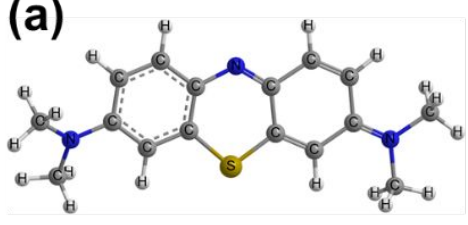

$1.8 \times 5.5 \times 14.2$

Methylene blue (+1)

(d)

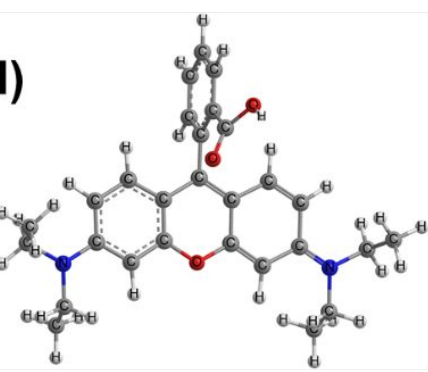

$15.6 \times 13.5 \times 4.2$

Rhodamine B (+1) (b)

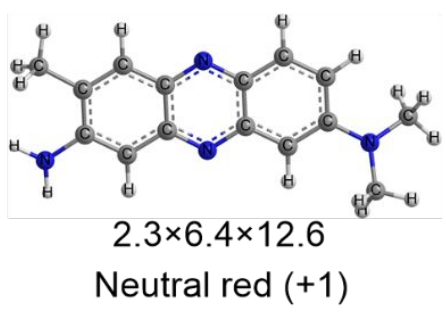

(e)

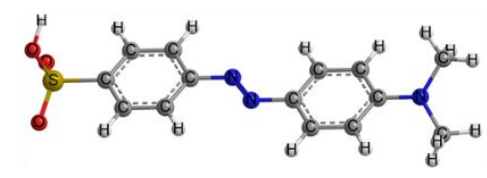

$4.5 \times 6.0 \times 14.8$

Methyl orange (-1)

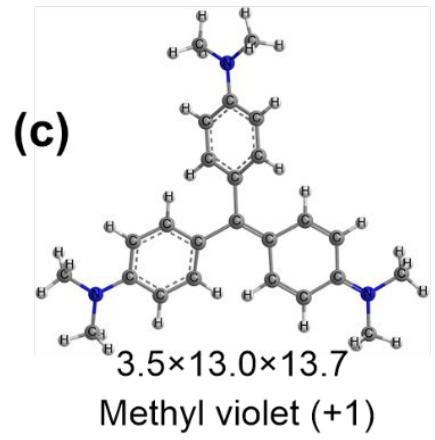

(f)

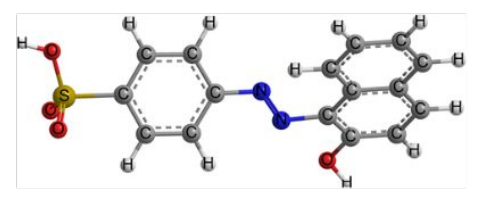

$13.5 \times 7.2 \times 2.8$

Orange II (-1)

Figure S1. The structures of the dyes used in the text. 
(a)

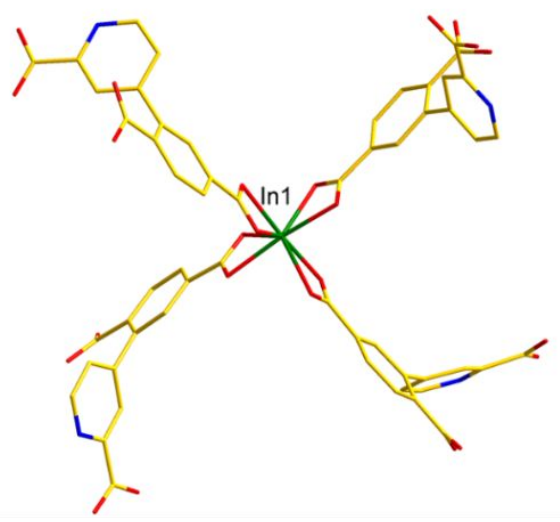

(b)

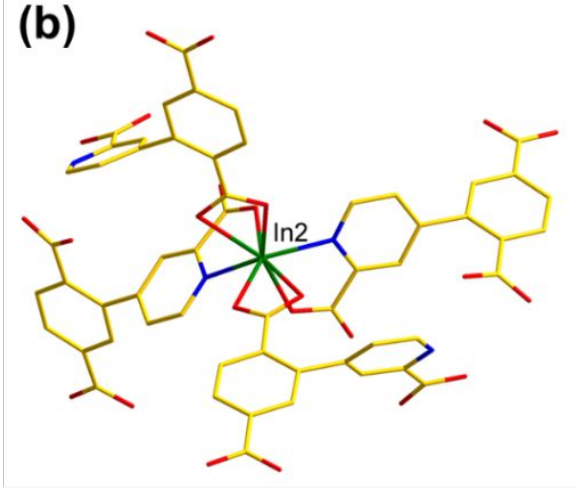

Figure S2. (a) Chemical environment of $\ln 1$ and (b) $\ln 2$ units.
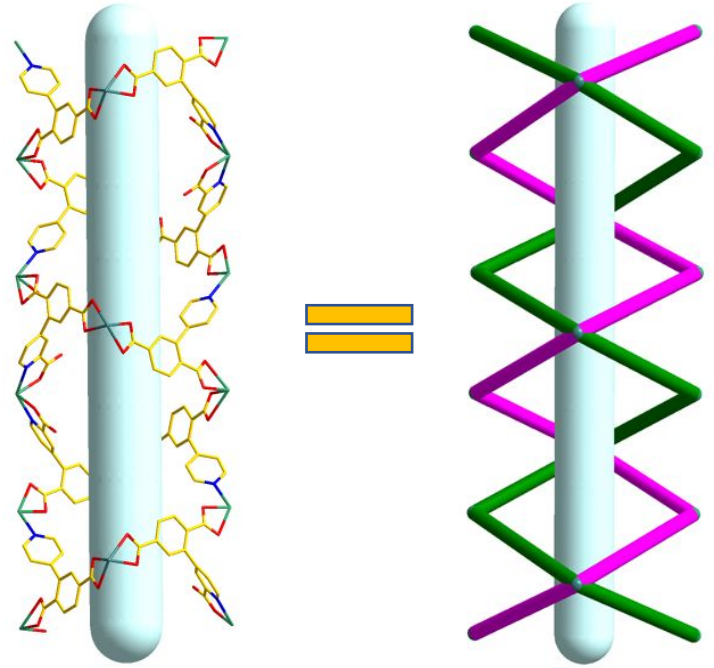

Figure S3. Two infinitely extending in- $L$ and $-R$ spiral chains from the $b$-axis direction

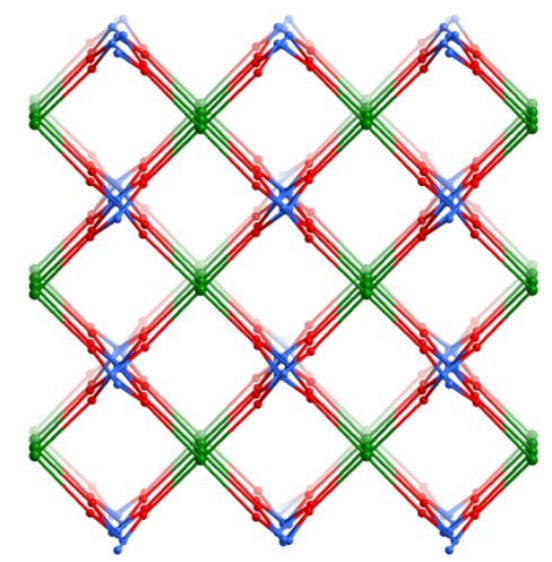

Figure S4. The $(3,4,4)$-connected topology of 1 . CPTA ${ }^{3-}$ ligands are red balls, $\ln 1$ atoms are green balls, and $\ln 2$ atoms are blue balls. 


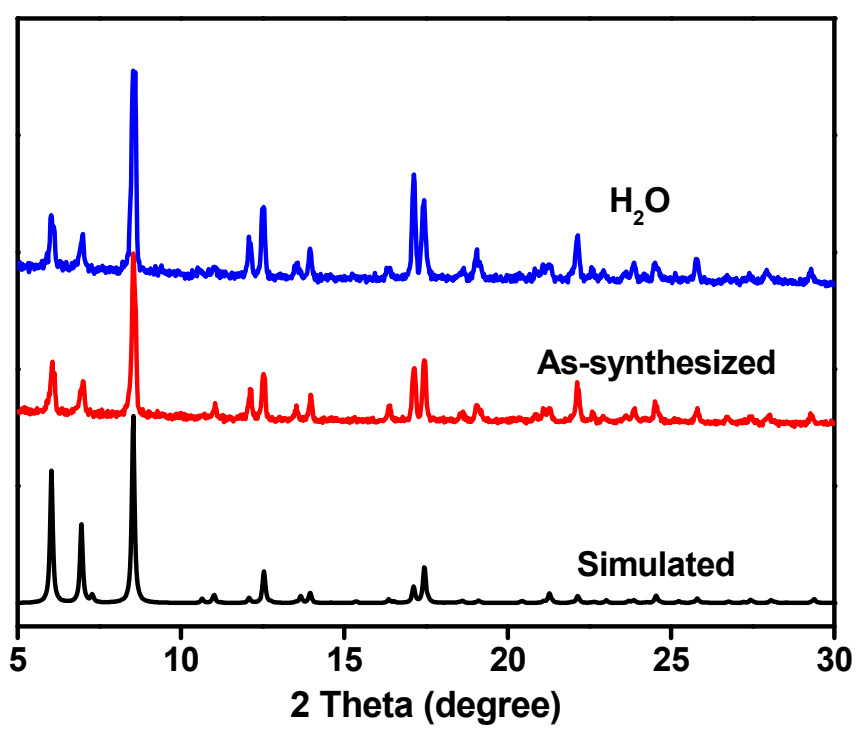

Figure S5. PXRD pattern of 1 simulated from the X-ray single-crystal structure, as-synthesized and 1 after soaking in water for $24 \mathrm{~h}$.

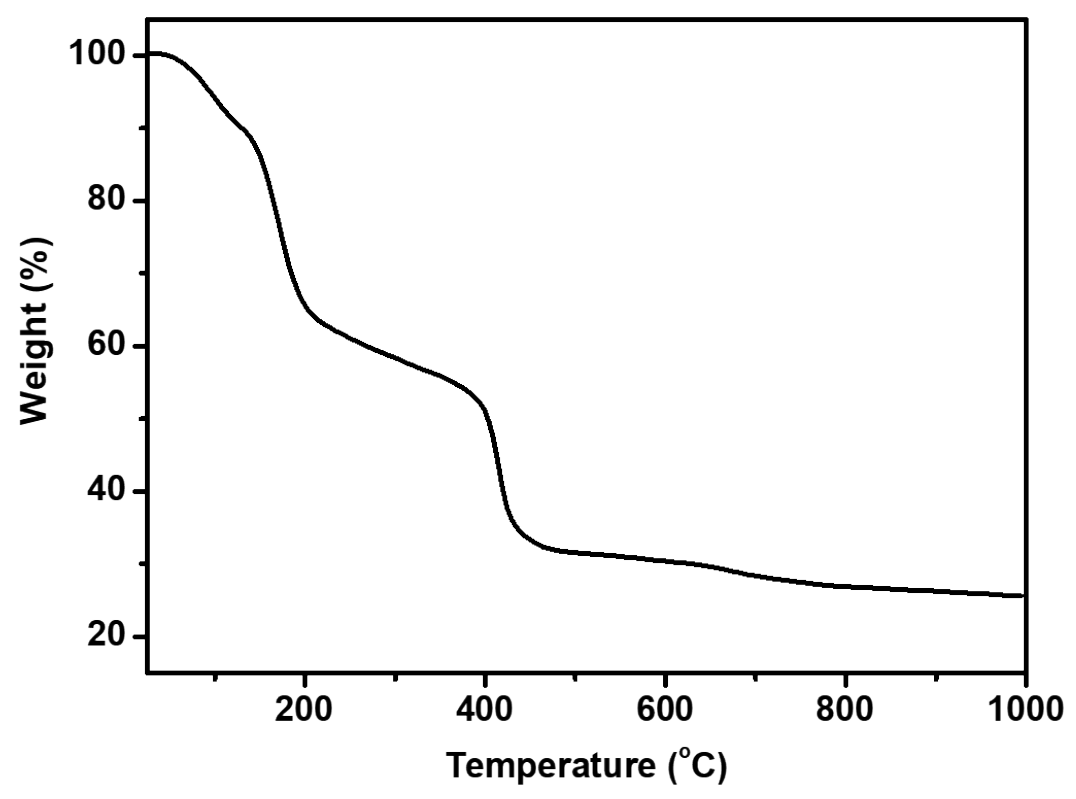

Figure S6. TGA curve for as-synthesized of 1. 


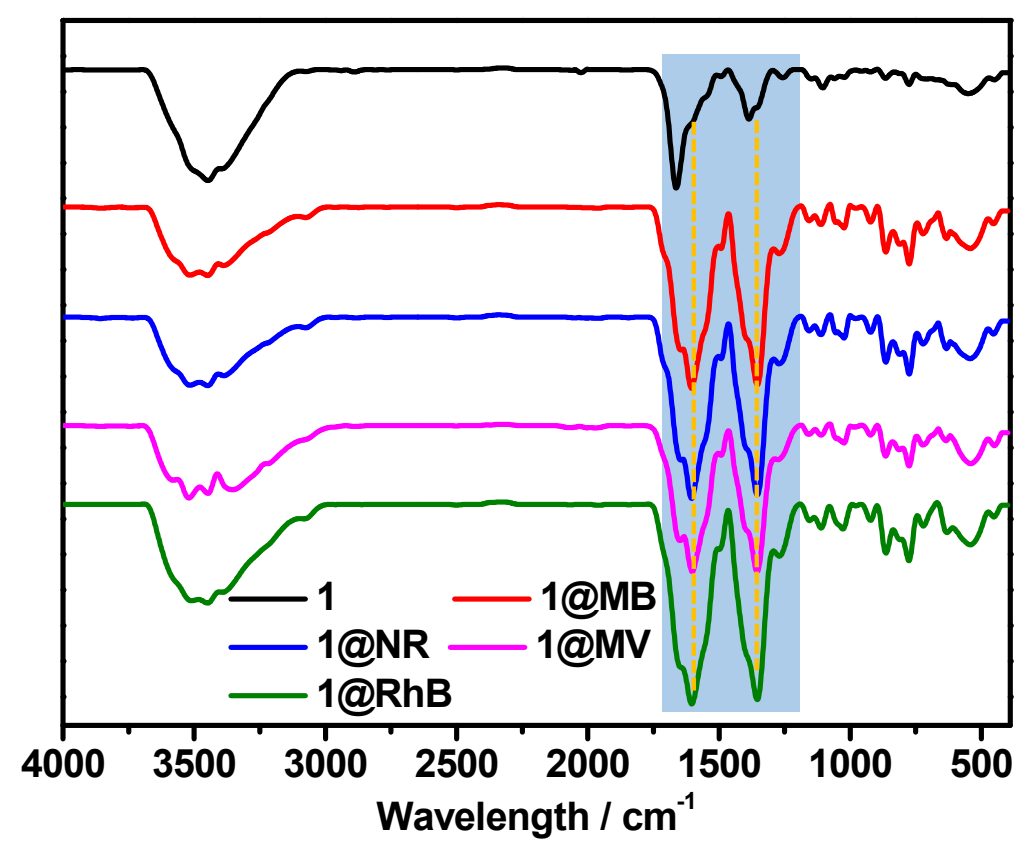

Figure S7. IR spectra of 1 for the as-synthesized, MB, NR, MV and RhB adsorbed states.

(a)
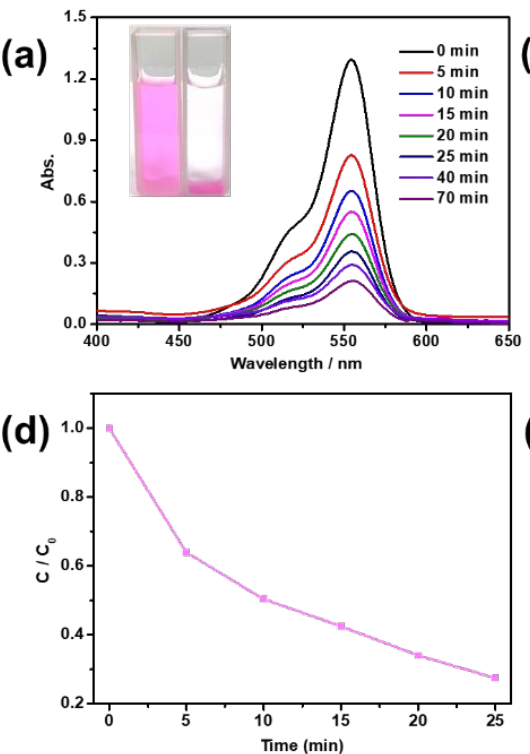
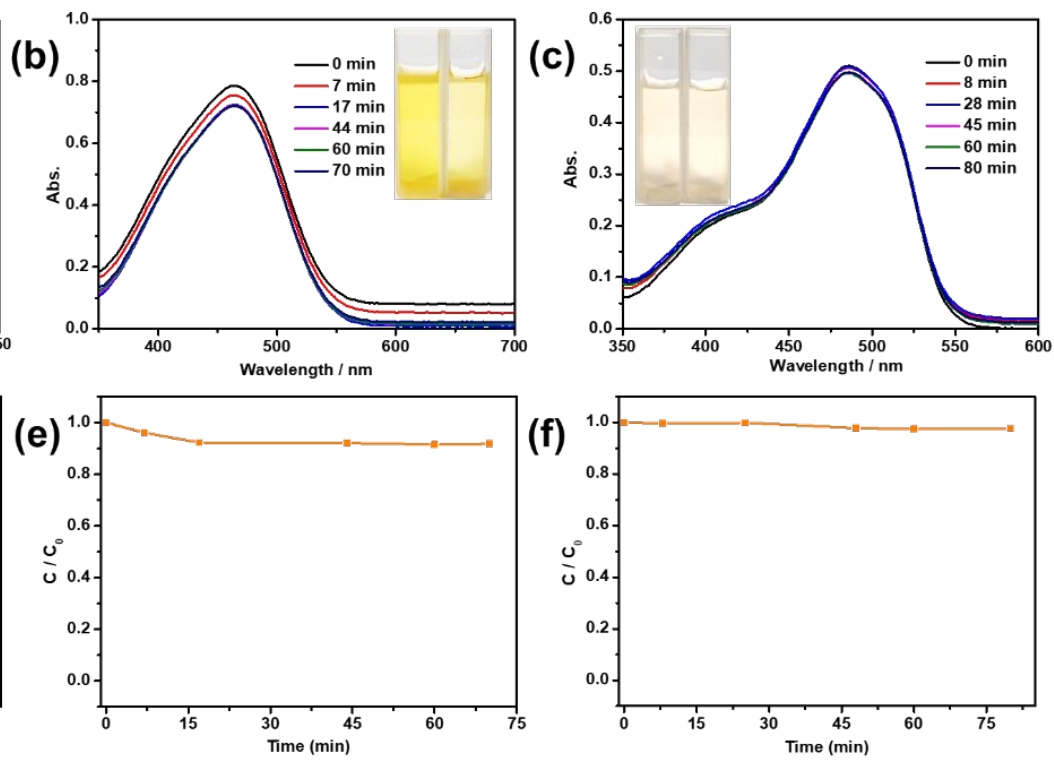

(f)

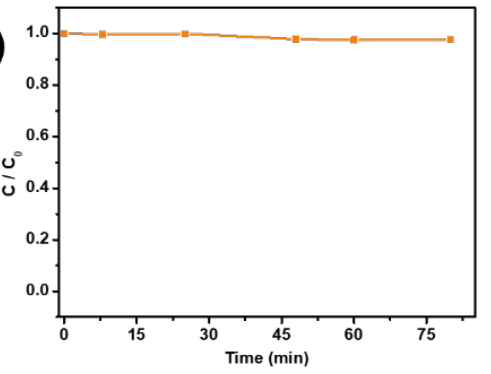

Figure S8. UV-vis absorption spectrum of aqueous solutions of RhB (a), MO (b) and MV (c); the adsorption rate of RhB (d), MO (e) and MV (f) in the presence of 1 , respectively. 


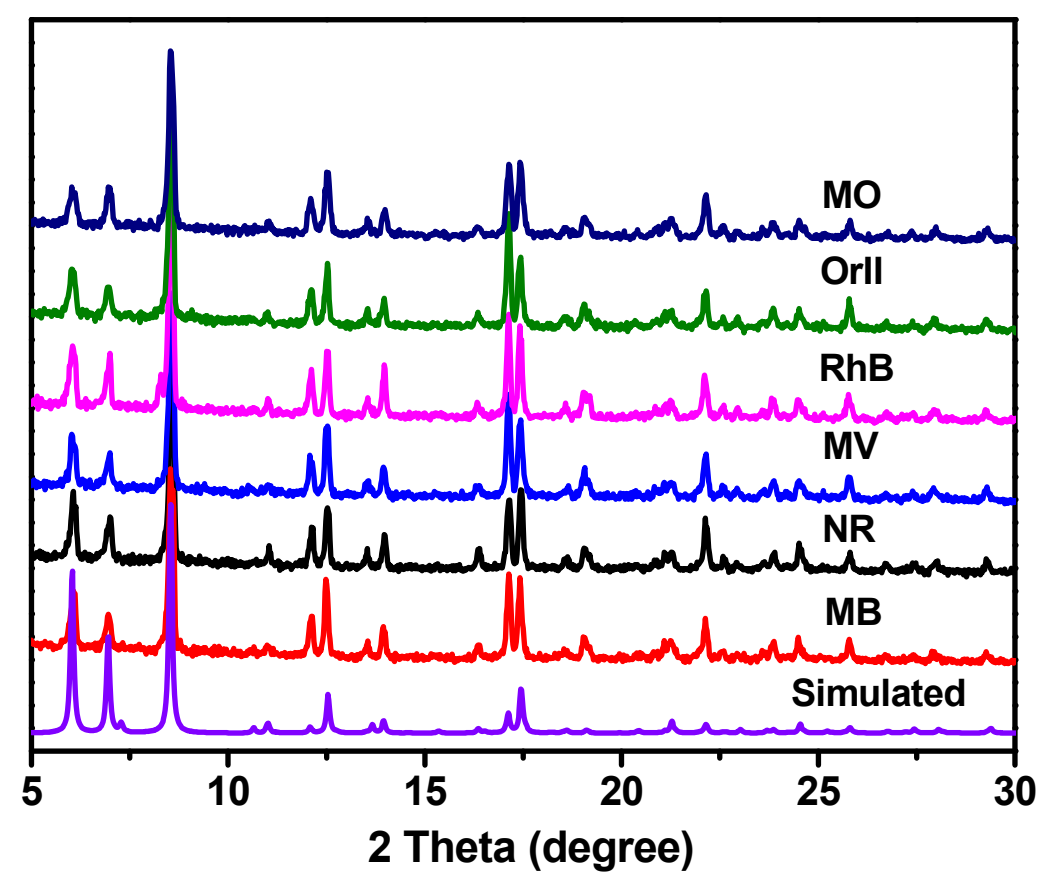

Figure S9. PXRD patterns after different dyes adsorption.

(a)

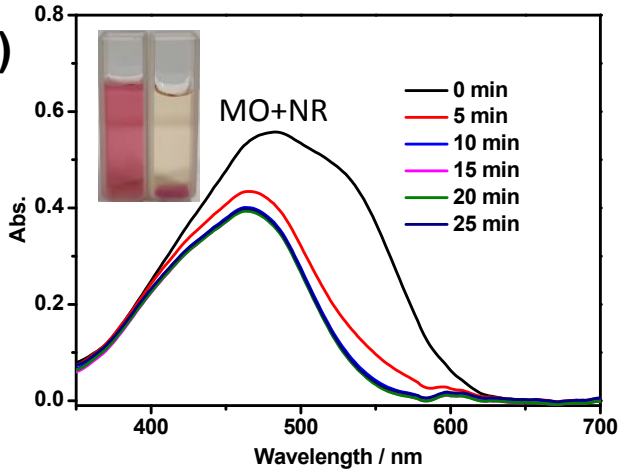

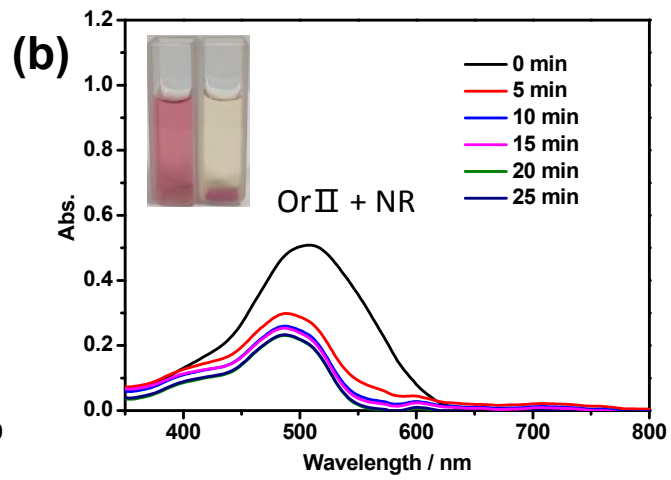

Figure S10. (a) and (b) are UV-vis spectrum of NR + MO and NR + Orll mixed aqueous solution after the addition of $\mathbf{1}$, respectively. 


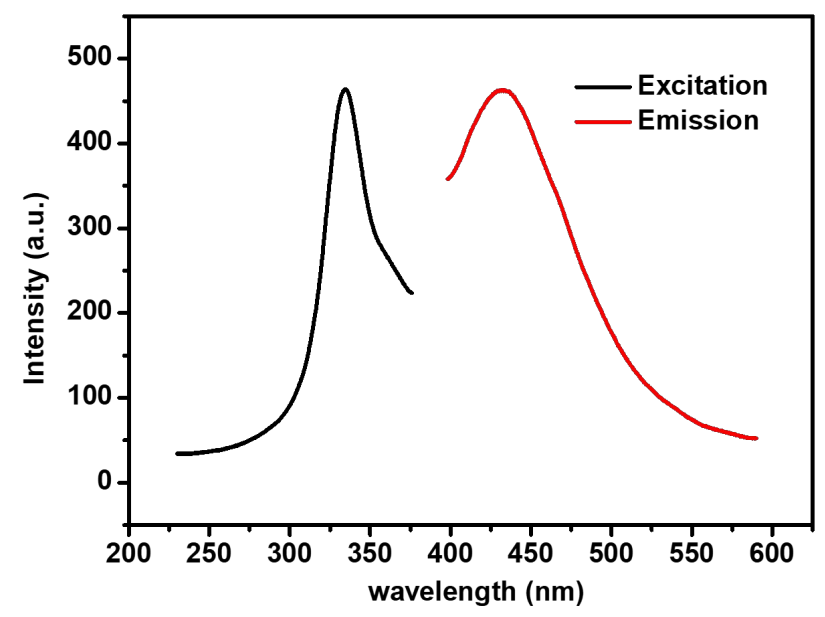

Figure S11. Excitation and emission spectra of 1.

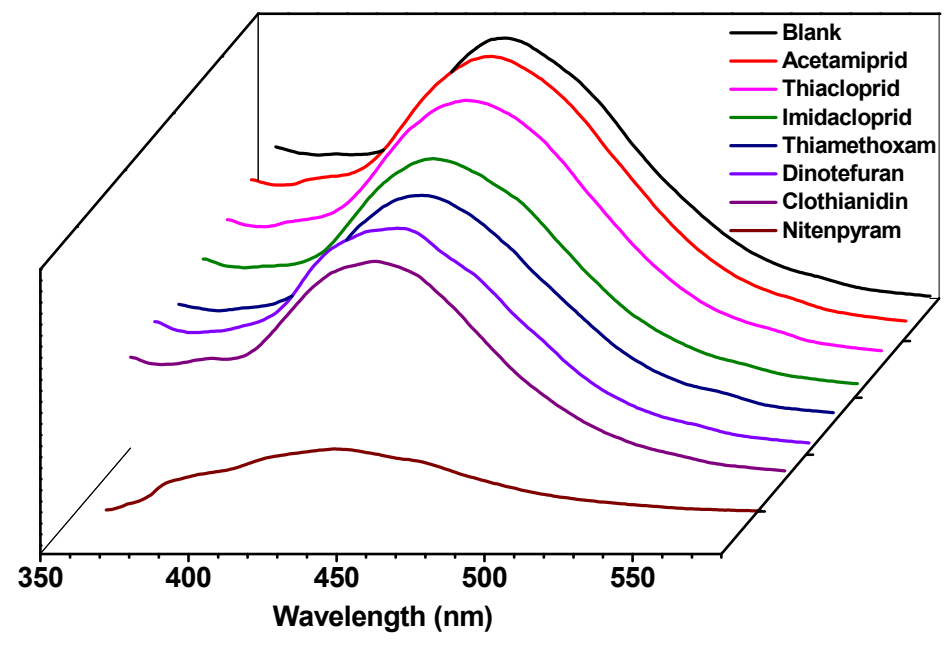

Figure S12. Fluorescence intensity of 1 in different neonicotinoid insecticide solutions.

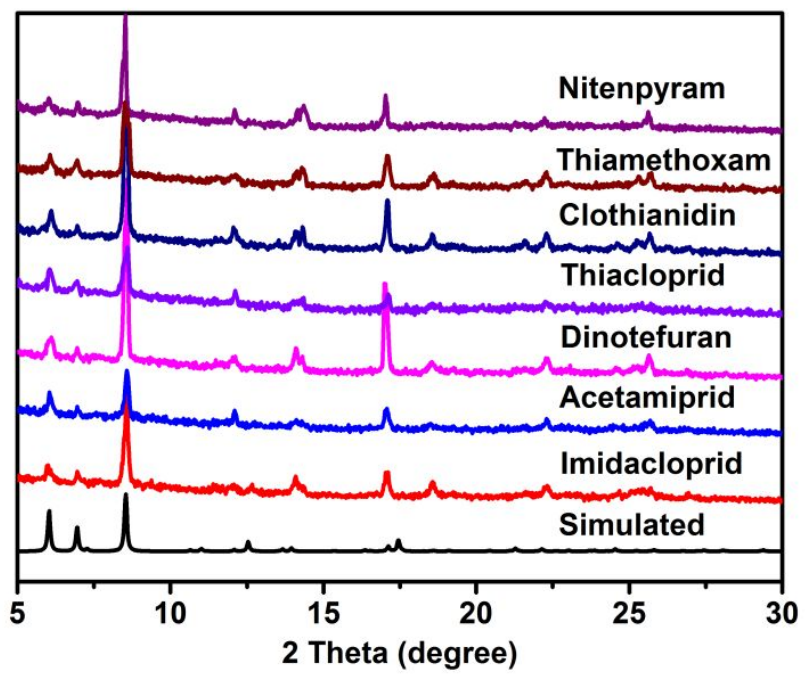

Figure S13. PXRD patterns after different neonicotinoid pesticide treatments. 
(a)

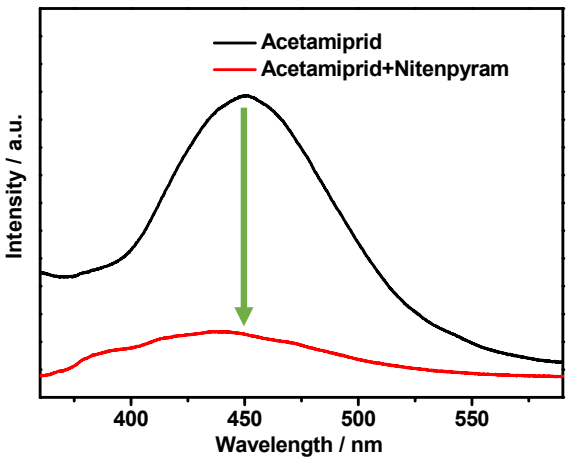

(c)

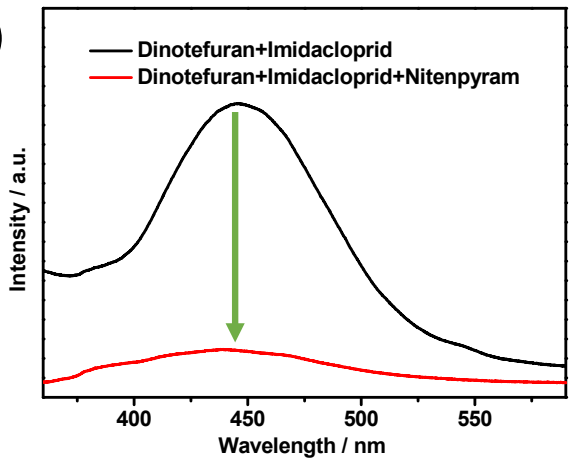

(b)

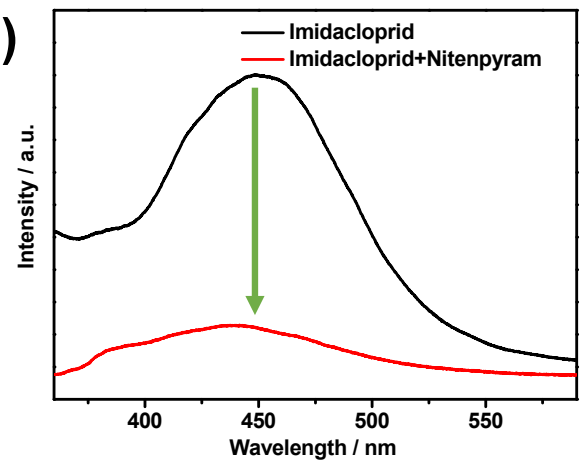

(d)

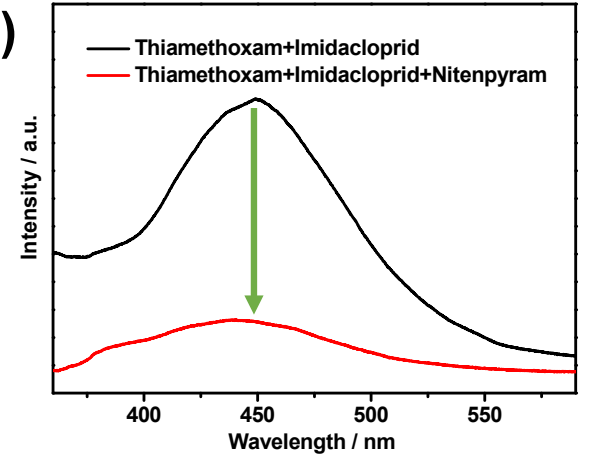

Figure S14. Selectivity of nitenpyram in the presence of different neonicotinoid pesticide.

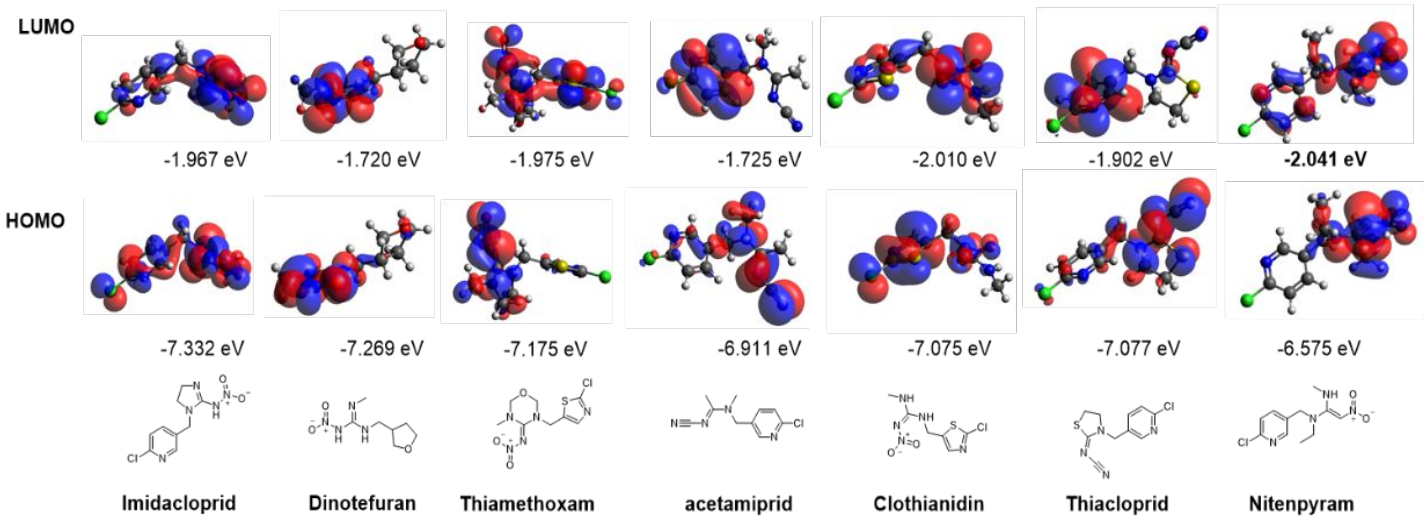

Figure S15. The orbital diagram of pesticide molecules. 


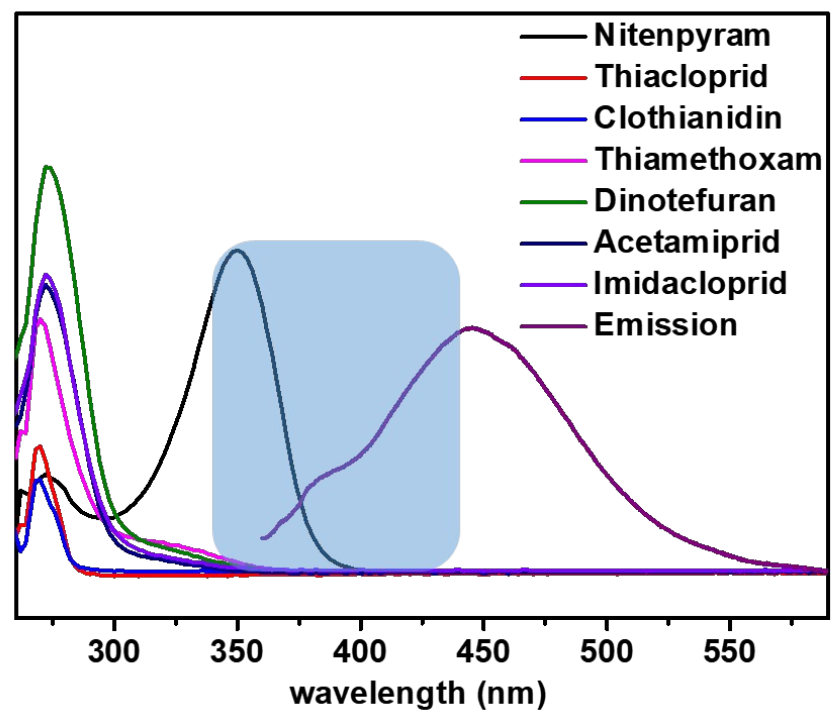

Figure S16. Absorption spectra of pesticides and emission spectra of 1.

Table S1 Partial Bond Length and Bond Angle Table of 1.

\begin{tabular}{|c|c|c|c|}
\hline In1 O2 & $2.207(4)$ & $\ln 2 \mathrm{O}^{4}$ & $2.194(4)$ \\
\hline $\ln 1 \mathrm{O} 2^{1}$ & $2.207(4)$ & $\ln 204^{5}$ & $2.194(4)$ \\
\hline $\ln 1 \mathrm{O}^{2}$ & $2.207(4)$ & $\ln 205^{4}$ & $2.555(4)$ \\
\hline $\ln 102^{3}$ & $2.207(4)$ & $\ln 2010^{6}$ & $2.197(3)$ \\
\hline $\ln 103$ & $2.334(3)$ & $\ln 2010$ & $2.197(3)$ \\
\hline $\ln 1 \mathrm{O}^{3}$ & $2.334(3)$ & $\ln 205^{5}$ & $2.255(4)$ \\
\hline $\ln 103^{1}$ & $2.334(3)$ & $\ln 2 \mathrm{~N} 1^{6}$ & $2.274(4)$ \\
\hline $\ln 1 \mathrm{O}^{2}$ & $2.334(3)$ & $\ln 2 \mathrm{~N} 1$ & $2.274(4)$ \\
\hline $\mathrm{O} 2 \ln 1 \mathrm{O} 2^{1}$ & $90.77(2)$ & $O 4^{5} \ln 205^{5}$ & $53.96(12)$ \\
\hline $\mathrm{O} 2^{1} \ln 1 \mathrm{O} 2^{2}$ & $90.78(2)$ & $\mathrm{O} 4^{4} \ln 205^{4}$ & $53.96(12)$ \\
\hline $\mathrm{O} 2 \ln 1 \mathrm{O}^{2}$ & 166.64(19) & $\mathrm{O} 4^{5} \ln 2 \mathrm{O} 10$ & $87.75(12)$ \\
\hline $\mathrm{O} 2 \ln 1 \mathrm{O}^{2}$ & $90.78(2)$ & $O 4^{4} \ln 2010^{6}$ & $87.75(12)$ \\
\hline $\mathrm{O} 2^{1} \ln 1 \mathrm{O} 2^{2}$ & 166.64(19) & $O 4^{5} \ln 2010^{6}$ & $151.57(13)$ \\
\hline $\mathrm{O} 2^{3} \ln 1 \mathrm{O} 2^{2}$ & $90.78(2)$ & $\mathrm{O} 4^{4} \ln 2010$ & $151.57(13)$ \\
\hline $\mathrm{O} 2^{3} \ln 1 \mathrm{O} 3^{3}$ & $57.45(12)$ & $\mathrm{O} 4^{4} \ln 2 \mathrm{~N} 1$ & $82.40(13)$ \\
\hline
\end{tabular}


$\mathrm{O} 2^{2} \ln 1 \mathrm{O}^{1}$

$85.43(12)$

$\mathrm{O} 4^{4} \ln 2 \mathrm{~N} 1^{6}$

$129.11(13)$

Symmetry transformations used to generate equivalent atoms:

13/4-Y,-1/4+X,5/4-Z; 21/4+Y,3/4-X,5/4-Z; ${ }^{31} 1-X, 1 / 2-Y,+Z ; ~ 43 / 4-Y, 1 / 4+X,-1 / 4+Z ;$

$5-1 / 4+Y, 1 / 4+X, 5 / 4-Z ; 61 / 2-X,+Y, 1-Z$

Table S2 the maximum adsorption amounts for MB, NR and MV on 1, based on the data of different initial concentrations experiments.

\begin{tabular}{c|cccccc} 
Dyes & $\mathbf{1}$ & $\mathbf{2}$ & $\mathbf{3}$ & $\mathbf{4}$ & $\mathbf{5}$ & Average $(\mathbf{m g} \mathbf{g})$ \\
\hline $\boldsymbol{M V}$ & 205 & 206 & 205 & 196 & 202 & $\mathbf{2 0 2 . 8}$ \\
$\mathbf{N R}$ & 167 & 170 & 173 & 168 & 176 & $\mathbf{1 7 0 . 8}$ \\
$\mathbf{M B}$ & 170 & 167 & 170 & 175 & 165 & $\mathbf{1 6 9 . 4}$
\end{tabular}

Table S3 Comparison of maximum adsorption amounts for MB.

\begin{tabular}{cccc}
\hline adsorbents & $\mathrm{Q}_{\mathrm{e}}(\mathrm{mg} / \mathrm{g})$ & Solvent & Ref. \\
\hline MOF-235 & 187 & $\mathrm{H}_{2} \mathrm{O}$ & 1 \\
MOF 1 & 105 & $\mathrm{H}_{2} \mathrm{O}$ & 2 \\
Ni-MOF & 156 & $\mathrm{H}_{2} \mathrm{O}$ & 3 \\
MIL-101(Al) & 195 & $\mathrm{H}_{2} \mathrm{O}$ & 4 \\
Cu-BTC & 200 & $\mathrm{H}_{2} \mathrm{O}$ & 5 \\
CPM-97-Fe & 304 & $\mathrm{H}_{2} \mathrm{O}$ & 6 \\
Sr-BTTC & 270 & DMA & 7 \\
$\mathbf{1}$ & 169.4 & $\mathrm{H}_{2} \mathrm{O}$ & this work \\
\hline
\end{tabular}

Table S4 Comparison of maximum adsorption amounts for MV.

\begin{tabular}{cccc}
\hline adsorbents & $\mathrm{Q}_{\mathrm{e}}(\mathrm{mg} / \mathrm{g})$ & Solvent & Ref. \\
\hline JLU-MONT1 & 1615 & DMF & 8 \\
HPP-3 & 699 & $\mathrm{H}_{2} \mathrm{O}$ & 9 \\
$\mathbf{1}$ & 202.8 & $\mathrm{H}_{2} \mathrm{O}$ & this work \\
\hline
\end{tabular}




\begin{tabular}{cccc}
\hline Sr-BTTC & 184 & $\mathrm{H}_{2} \mathrm{O}$ & 7 \\
CSAC & 135.1 & $\mathrm{H}_{2} \mathrm{O}$ & 10 \\
TPR & 94.3 & $\mathrm{H}_{2} \mathrm{O}$ & 11 \\
JLU-Liu39 & 84 & $\mathrm{EtOH}$ & 12 \\
Magnetic nanocomposite & 81.7 & $\mathrm{H}_{2} \mathrm{O}$ & 13 \\
\hline
\end{tabular}

Table S5 Standard Deviation $(\sigma)$ calculation for the detection of nitenpyram for 1

\begin{tabular}{cc} 
Test & Fluorescence Intensity \\
1 & 377 \\
2 & 380 \\
3 & 384 \\
4 & 370 \\
5 & 383 \\
6 & 370 \\
average & 377.33 \\
ard Deviation $(\sigma)$ & 5.65 \\
\hline
\end{tabular}

\section{Reference:}

(1) Haque, E.; Jun, J. W.; Jhung, S. H. Adsorptive removal of methyl orange and methylene blue from aqueous solution with a metal-organic framework material, iron terephthalate (MOF-235). J. Hazard. Mater. 2011, 185 (1), 507-511.

(2) Yi, F.-Y.; Li, J.-P.; Wu, D.; Sun, Z.-M. A Series of Multifunctional MetalOrganic Frameworks Showing Excellent Luminescent Sensing, Sensitization, and Adsorbent Abilities. Chem. Eur. J. 2015, 21 (32), 11475-11482.

(3) Zhao, S.; Chen, D.; Wei, F.; Chen, N.; Liang, Z.; Luo, Y. Synthesis of graphene oxide/metal-organic frameworks hybrid materials for enhanced 
removal of Methylene blue in acidic and alkaline solutions. J. Chem. Technol. Biotechnol. 2018, 93 (3), 698-709.

(4) Haque, E.; Lo, V.; Minett, A. I.; Harris, A. T.; Church, T. L. Dichotomous adsorption behaviour of dyes on an amino-functionalised metal-organic framework, amino-MIL-101(Al). J. Mater. Chem. A 2014, 2 (1), 193-203.

(5) Lin, S.; Song, Z.; Che, G.; Ren, A.; Li, P.; Liu, C.; Zhang, J. Adsorption behavior of metal-organic frameworks for methylene blue from aqueous solution. Microporous Mesoporous Mater. 2014, 193, 27-34.

(6) Tian, S.; Xu, S.; Liu, J.; He, C.; Xiong, Y.; Feng, P. Highly efficient removal of both cationic and anionic dyes from wastewater with a water-stable and eco-friendly Fe-MOF via host-guest encapsulation. J. Clean. Prod. 2019, 239, 117767.

(7) Shen, Y.; Fan, C.-C.; Wei, Y.-Z.; Du, J.; Zhu, H.-B.; Zhao, Y. An anionic zeolite-like metal-organic framework (AZMOF) with a Moravia network for organic dye absorption through cation-exchange. Dalton Trans. 2016, 45 (27), 10909-10915.

(8) Zhou, Y.; Yao, S.; Ma, Y.; Li, G.; Huo, Q.; Liu, Y. An anionic single-walled metal-organic nanotube with an armchair $(3,3)$ topology as an extremely smart adsorbent for the effective and selective adsorption of cationic carcinogenic dyes. Chem. Commun. 2018, 54 (24), 3006-3009.

(9) Liu, H.; Liu, H. Selective dye adsorption and metal ion detection using multifunctional silsesquioxane-based tetraphenylethene-linked nanoporous polymers. J. Mater. Chem. A 2017, 5 (19), 9156-9162.

(10) Özdemir, M.; Durmuş, Ö.; Şahin, Ö.; Saka, C. Removal of methylene blue, methyl violet, rhodamine B, alizarin red, and bromocresol green dyes from aqueous solutions on activated cotton stalks. Desalin. Water Treat. 2016, 57 (38), 18038-18048.

(11) Kannan, C.; Buvaneswari, N.; Palvannan, T. Removal of plant poisoning dyes by adsorption on Tomato Plant Root and green carbon from aqueous solution and its recovery. Desalination 2009, 249 (3), 1132-1138. 
(12) Yao, S.; Xu, T.; Zhao, N.; Zhang, L.; Huo, Q.; Liu, Y. An anionic metalorganic framework with ternary building units for rapid and selective adsorption of dyes. Dalton Trans. 2017, 46 (10), 3332-3337.

(13) Singh, K. P.; Gupta, S.; Singh, A. K.; Sinha, S. Optimizing adsorption of crystal violet dye from water by magnetic nanocomposite using response surface modeling approach. J. Hazard. Mater. 2011, 186 (2), 1462-1473. 